\title{
Performance Evaluation of Adaptive Modulation Based MC-CDMA System
}

\author{
Iftekhar Alam ${ }^{1}$, Vikas Srivastva1, Arun Prakash ${ }^{2}$, Rajeev Tripathi ${ }^{2}$, A. K. Shankhwar ${ }^{3}$ \\ ${ }^{1}$ Wireless Communication and Computing, IIIT Allahabad, India; ${ }^{2}$ ECED, MNNIT, Allahabad, India; ${ }^{3}$ Electronics Engineering, \\ HBTI, Kanpur, India. \\ Email: iwc2011023@iiita.ac.in
}

Received September $25^{\text {th }}, 2012$; revised October $29^{\text {th }}, 2012$; accepted November $28^{\text {th }}, 2012$

\begin{abstract}
In today's scenario mobile phones are not used only for messaging and talking, now we use mobiles also to access internet. For that we need higher data rates. Mobiles use wireless communication channel, which is very fast varying channel. To increase the data rate we have to utilize the full channel based on the channel condition. For that we have to estimate the channel condition and based on that we have to vary the data rate so that our communication system performance parameters (bit error rate, throughput) do not change beyond the accepted level. To increase the data rate we are also using the multi-carrier CDMA system in conjunction with the above described adaptive modulation. Adaptive modulation, in conjunction with MC-CDMA transmission system is very assuring way to increase data rate. In current scenario demand of very high data rates up to $100 \mathrm{Mbits} / \mathrm{s}$ may be achieved with this scheme. In this paper, adaptive modulation based M-ary QAM, M-PSK and M-ary CPM systems applied to a MC-CDMA system in Rayleigh fading channel environment have been investigated and BER performances of all these digital modulation schemes have been compared.
\end{abstract}

Keywords: Adaptive Modulation; CDMA; Digital Modulation; MC-CDMA; OFDM

\section{Introduction}

In wireless communication system spectrum bandwidth is the most precious commodity. The main problem to the wireless channel is Rayleigh fading channel that degrades the error performance of the wireless communication system. In wireless communication channel the signal to noise ratio (SNR) is time variant due to multipath fading and interference from other users, in direct contrast with the additive white Gaussian noise (AWGN) channel which is characterized by a constant signal to noise ratio.

We may utilize full channel capacity in two ways in presence of multipath fading and interference from other users: adaptation and diversity techniques. By adaptation we mean to change the modulation scheme and/or coding used by transmitter according to the channel condition.

Figure 1 shows that the channel estimation is needed for using different digital modulation techniques. As we increase the range of communication, we step down to lower modulations but as we reduce the communication range means we are closer to the base station we can utilize higher order modulations for increased throughput. Adaptive modulation also allows the system to overcome multipath fading and other interference. In essence, this is a way to optimize the modulation scheme for the better transmission of data at higher rate in accordance to the state of the channel for required fidelity. For example, when the channel condition is poor we may reduce the modulation index in order to improve fidelity. Conversely if the channel is in good condition we can increase the modulation index to increase the data rate.

The remainder of the paper is organized as follows. Author presents survey of some relevant adaptive modulation schemes in Section 2, followed by the system model discussed in Section 3 in which author presents a model and some formulas for calculating SNR. The paper continues with result and discussion in Section 4. Finally, the paper is concluded.

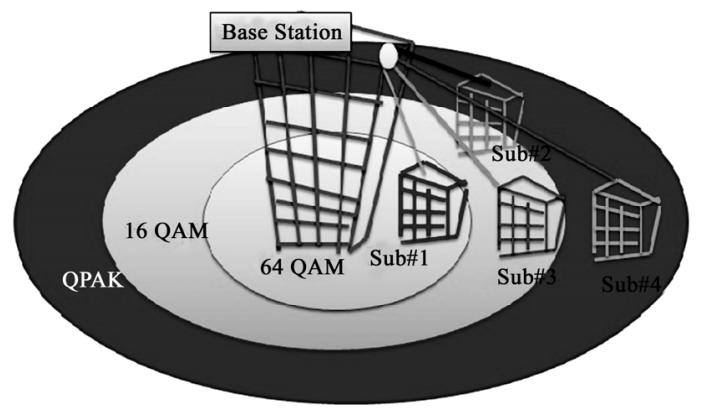

Figure 1. Illustration of adaptive modulation. 


\section{Related Work}

Adaptive technology has been firstly investigated in late 60 's and early 70 's $[1,2]$. Interest in this modulation scheme was short lived, because of hardware constraints and lack of good channel estimation techniques. As technology improved these issues became less constraining, which resulted in a revived interest of researchers in adaptive modulation schemes for 3 rd generation wireless systems [3].

In the adaptive techniques, transmission parameters, for example transmission power [4], symbol transmission rate [5], constellation size [6-8], coding rate/scheme [9], or any combination of these parameters $[8,10]$, are changed in response to the estimated time-varying channel conditions. Many researchers have given their system model by using different combination of modulation schemes and evaluate its performance in terms of BER, throughput and data rate and showed improvement from the existing systems. Some of them we studies are:

\subsection{Reliable Adaptive Modulation Aided by Observations of Another Fading Channel}

Tung-Sheng Yang and Alexandra Duel-Hallen in [11] have proposed an approach to use adaptive transmission technique using past channel observations of 1 carrier to predict future channel state information and this technique performs adaptive modulation without feedback path for another correlated carrier. They derived a MMSE (Minimum mean-square error) long range channel prediction method which uses the time-domain and frequency domain correlation function of Rayleigh fading channel. They used a standard stationary fading channel model to test their algorithm and showed a significant gain over non adaptive techniques.

If the communication between 2 stations is bidirectional then each station can estimate the channel condition and adapt the parameters to this estimation. This is known as open-loop adaptation [12]. And if the communication between two stations is not bidirectional the receiver has to estimate channel condition and feedback it to the transmitter, resulting in closed loop adaptation. The feed back load of the channel estimation information consumes system power and bandwidth, resulting in degraded performance. For many adaptive transmission applications, it is not necessary to feed back the actual fading coefficient instead of this it is sufficient to feed back to the transmitter only the antenna selection or modulation index produced from the estimated or predicted values at the receiver [13]. The feedback delay, channel estimation and processing delay degrade the performance of this adaptive modulation technique, especially in rapidly time-variant fading. To realize the good performance improvement for adaptive transmis- sion techniques, the channel variations should be predicted reliably at least several milliseconds ahead. They extended the LRP algorithm into frequency domain. This LRP method was proposed in $[14,15]$. This long range prediction (LRP) algorithm utilizes an autoregressive model for characterizing the fading channel and also computes the minimum mean square error to estimate the future fading coefficient on the basis of past observations. Particularly the concentrated on a scenario where they observed a received uplink signal at the carrier of frequency $\mathrm{f}^{\wedge} 1$ and attempted to predict the down link signal at carrier of frequency $\mathrm{f}^{\wedge} 2$ without any feedback from the mobile. For the accomplishment of this prediction, the predicted samples should be sufficiently correlated in both frequency and time.

\subsection{Adaptive Modulation Systems for Predicted Wireless Channel}

Sorour Falahati and Arne Svensson have studied an optimum design for an adaptive modulation technique based on encoded M-QAM, assisted with channel prediction for the Rayleigh fading channel in [15]. Signaling delays create problem with outdated Channel State Information (CSI) in adaptive modulation. This degrades the adaptive modulation system performance such as decrease in the throughput. The use of power prediction will improve the performance of adaptive modulation. Sorour Falahati and Arne Svensson have shown their interest to take the quality of these predictions into account in designing adaptive modulation scheme. The key issues they studied in [15] are how a known prediction error variance can affect the transmission properties, such as the signal-to-noise ratio (SNR) boundaries that determine when to change the modulation index, and to what extent it could affect the spectral efficiency. Figure 2 shows the discrete model of the system they proposed. They considered fast link adaptation where the receiver estimates the received power and transfers this information to the transmitter site via feedback channel to modify the transmission parameters such as modulation index, estimates of the channel state information will be based on outdated information. Theoretically an optimized way of handling this problem could be to estimate the conditional probability density

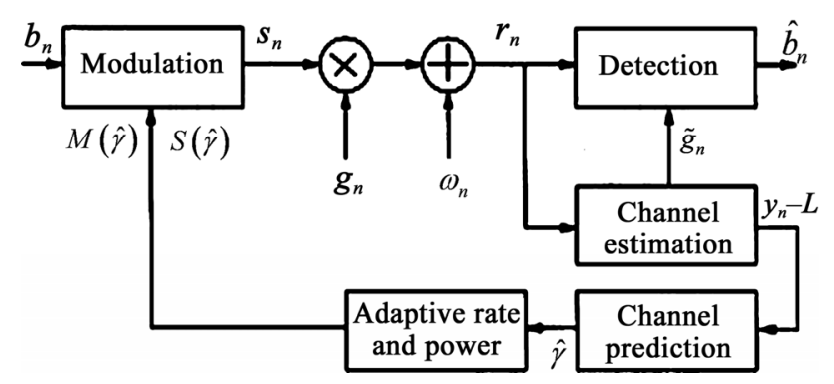

Figure 2. Discrete model of the system. 
function of the BER optimally for a given modulation format form the all past received information signals and other relevant information. And then the modulation scheme is selected on the basis of this probability density function. But this proposed approach is hard to realize because the BER is a non-linear function of channel power gain. Therefore they considered a suboptimal approach that the channel power gain is predicted by a scheme and adaptive modulation system is adjusted on the basis of statistics of these predictions.

\section{System Model}

\subsection{System Model and Block Diagram}

Figure 3 shows the block diagram for the adaptive modulation based MC-CDMA system. Firstly the binary information is encoded with turbo codes, which is followed by a serial to parallel converter to produce a data stream of low bit rate in order to avoid the inter-symbol interference.

Then each stream is modulated with by a suitable digital method, in this thesis work we used BPSK, QPSK, 16QAM, 64-QAM, M-ary MHPM etc., depending upon the current channel signal to noise ratio estimate information provided by the receiver. After this the modulated bit streams are passed through the Multi-carrier CDMA system transmitter then this is up-converted by an RF amplifier and finally transmitted through the channel. At the receiver site reverse operations are performed to demodulate and decode the original information. In this thesis we used pilot assisted channel estimation for estimating the quality of channel which estimates the carrier to noise ratio of the channel. Pilots symbols used are known QPSK symbols. The CDMA code used in this thesis is WalshHadamard code.

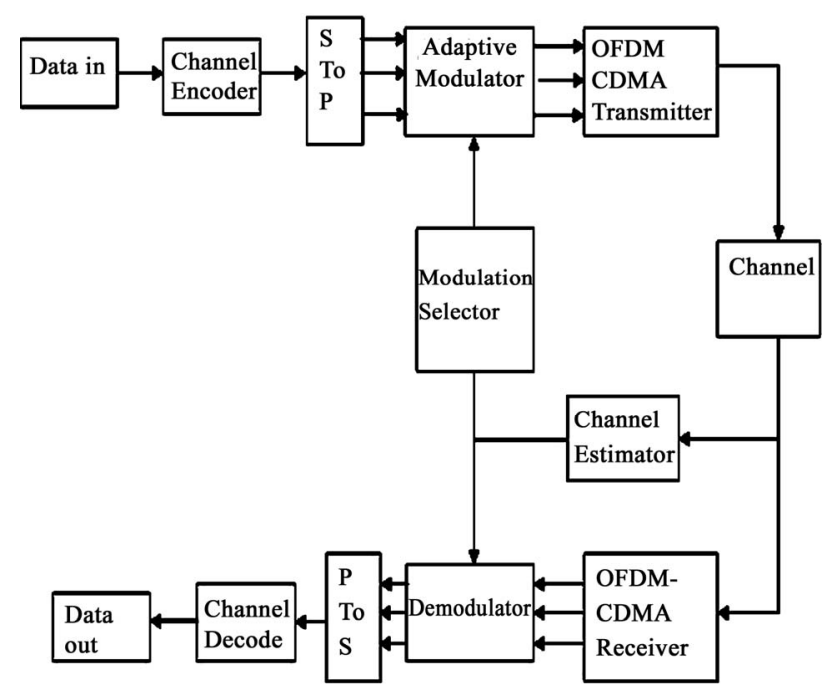

Figure 3. Block diagram for adaptive modulation base MCCDMA system.

\subsection{SNR Estimation}

The basic idea for channel estimation in our thesis is that if we have the signal which is corrupted by a given channel we can estimate SNR of the channel through mean and variance calculation. Firstly, the average received power is calculated by taking the square of mean of magnitude of the received signal. This will be used as estimated signal power. And the noise power will be calculated by variance calculation on the received signal.

For PSK modulation scheme, we use the following scheme to find the mean and variance of a currently received block of data:

$$
\begin{aligned}
& Z=\frac{1}{N} \sum_{i=1}^{N}\left|r_{i}\right| \\
& T^{2}=\frac{1}{N-1}\left(\sum_{i=1}^{N}\left(\left|r_{i}\right|-\left|c_{i}^{\prime}\right|\right)^{2}\right)
\end{aligned}
$$

where $Z$ represents mean of the received signal and $\mathrm{T}^{\wedge} 2$ represents the variance of the received signal corrupted though the channel noise $N$ represents the no of symbols over which SNR has to be estimated. $c_{-} i$ is the channel estimate coming from FFT estimator.

The SNR can be calculated as:

$$
\Gamma=\left(\frac{N-3}{N-1}\right) \frac{|Z|^{2}}{T^{2}}-\frac{1}{N}
$$

\subsection{Adaptive Modulation Boundaries}

In adaptive modulation system, we need a way to decide which modulation level is best suited for the present channel conditions. We decided to use the estimated SNR of the link as channel metric to decide switching levels. Pons and Dunlop in [16] proposed that bit error rate at the receiver would be really a good channel metric to decide modulation level switching. But our personnel experience says that BER estimation on small packet or for very short duration could not be reliably estimated.

Now the question arises how to decide that which modulation scheme is to be used for which range of SNR? Answer lies within the AWGN performance of every modulation scheme.

Received signal is given as $r(t)=c(t) \cdot s(t)+n(t)$. Where $c(t)$ is Rayleigh channel gain, $n(t)$ is additive Gaussian noise. In our system model we considered the signal power is to be the transmitted signal power multiplied with the Rayleigh channel gain. This signal power is the instantaneously received signal power, which could be directly compared to the Gaussian noise power. So, allowing us to consider the BER over an AWGN channel. Now consider the BER performance of the 3 modulation schemes used in our thesis. Form [17] equations for their BER are as follows: 


$$
\begin{aligned}
& P_{\mathrm{QPSK}}(Y)=Q(\sqrt{Y}) \\
& P_{16 \mathrm{QAM}}(Y)=\frac{1}{4}\left[Q\left(\sqrt{\frac{Y}{5}}\right)+Q\left(3 \sqrt{\frac{Y}{5}}\right)+\frac{1}{2} Q\left(\sqrt{\frac{Y}{5}}\right)\right] \\
& P_{64 \mathrm{QAM}}(Y)=\frac{1}{12}\left[Q\left(\sqrt{\frac{Y}{21}}\right)+Q\left(3 \sqrt{\frac{Y}{21}}\right)+Q\left(5 \sqrt{\frac{Y}{21}}\right)\right. \\
& +Q\left(7 \sqrt{\frac{Y}{21}}\right)+\frac{1}{6} Q\left(\sqrt{\frac{Y}{21}}\right)+\frac{1}{6} Q\left(3 \sqrt{\frac{Y}{21}}\right) \\
& +\frac{1}{6} Q\left(5 \sqrt{\frac{Y}{21}}\right)+\frac{1}{6} Q\left(7 \sqrt{\frac{Y}{21}}\right)+\frac{1}{3} Q\left(\sqrt{\frac{Y}{21}}\right) \\
& +\frac{1}{4} Q\left(3 \sqrt{\frac{Y}{21}}\right)-\frac{1}{4} Q\left(5 \sqrt{\frac{Y}{21}}\right)-\frac{1}{6} Q\left(7 \sqrt{\frac{Y}{21}}\right) \\
& +\frac{1}{6} Q\left(9 \sqrt{\frac{Y}{21}}\right)+\frac{1}{12}+Q\left(11 \sqrt{\frac{Y}{21}}\right) \\
& \left.-\frac{1}{12} Q\left(13 \sqrt{\frac{Y}{21}}\right)\right]
\end{aligned}
$$$$
P_{\mathrm{QPSX}}(Y)=Q 0
$$

In the above mentioned equations, $\gamma$ is the $\mathrm{SNR}, \& Q($. is the $Q$ function defined as:

$$
Q(x)=\frac{1}{\sqrt{2 \pi}} \int_{\gamma}^{N} \mathrm{e}^{-\frac{x^{2}}{2}} \mathrm{~d} x
$$

On the basis of above formula we calculate the BER and run the MATLAB simulation. We observe a considerable change in throughput and data rate in case of adaptive modulation. This is discussed in next section.

\section{Result and Discussion}

The MATLAB simulations of MC-CDMA in Rayleigh fading channel have been carried out and for various M-ary QAM, M-ary CPM, M-ary MHPM. Figure 4, the curves from right to left represent the BER for 64 QAM, 16 QAM and QPSK in a Rayleigh fading channel, respectively. For deciding the proper switching levels we need to decide first what performance, in terms of BER we need from our system. For our system model we decided to choose BER performance of $10^{\wedge}-3$ as operating point. This means that this system will try to keep the BER of the system lower than $10^{\wedge}-3$ with most spectrally efficient modulation scheme whenever possible. Spectral efficiency is defined as the number of information bits encoded on a single modulated transmission symbol.

Therefore, with our operating point, and given BER plots in Figure 4 we conclude to have the following SNR ranges for each modulation scheme:

\begin{tabular}{cc}
\hline QPSK & SNR $<16 \mathrm{~dB}$ \\
16 QAM & $17<=$ SNR $<=23 \mathrm{~dB}$ \\
64 QAM & SNR $>23 \mathrm{~dB}$ \\
\hline
\end{tabular}

We made this conclusion in the following way: from Figure 4, there is no modulation scheme that gives us performance below $10^{\wedge}-3$ at an SNR below $10 \mathrm{~dB}$. So, we choose QPSK as it was the most robust modulation scheme.

In the range of 10 to $17 \mathrm{~dB}$, there was only one scheme that gives us our desired performance that was QPSK. In the range of 17 to $23 \mathrm{~dB}, 16 \mathrm{QAM}$ gives us performance below $10^{\wedge}-3$, at a better spectral efficiency. And for SNR higher than $23 \mathrm{~dB}, 64$ QAM provides us our desired BER performance with the best spectral efficiency.

Figure 5 shows the BER performance under different channel SNR for a given number of users with M-ary MHPM as modulation technique. This figure also shows that the performance of the system degrades with the increase in the number of users due to multipath interference. The adaptive modulation with MC-CDMA technique gives a better BER performance as shown by green line.

\section{Conclusion and Future Scope}

In this paper we studied various adaptive modulated MC-CDMA systems in Rayleigh fading channel. The digital modulation schemes considered in our thesis are M-ary PSK, M-ary QAM, M-ary MHPM, M-ary CPM, and GMSK with varying bit-duration-bandwidth product. The results of our study of the adaptive modulated systems clearly show that the dynamic switching of the modulation orders can enhance the system performance and capacity per given bandwidth with the expected BER performance. With the adaptive modulation based systems,

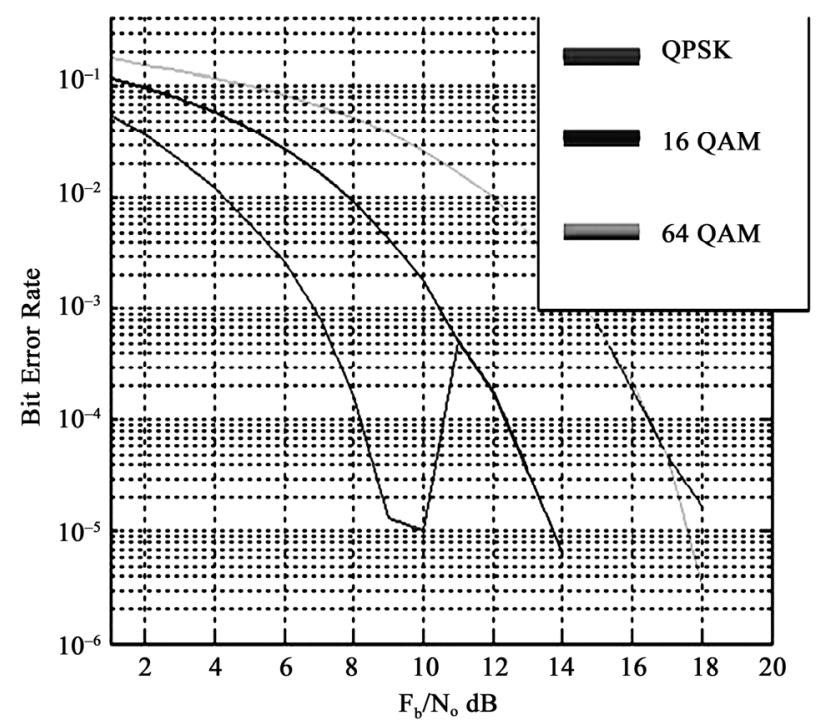

Figure 4. BER performances for different modulation scheme. 


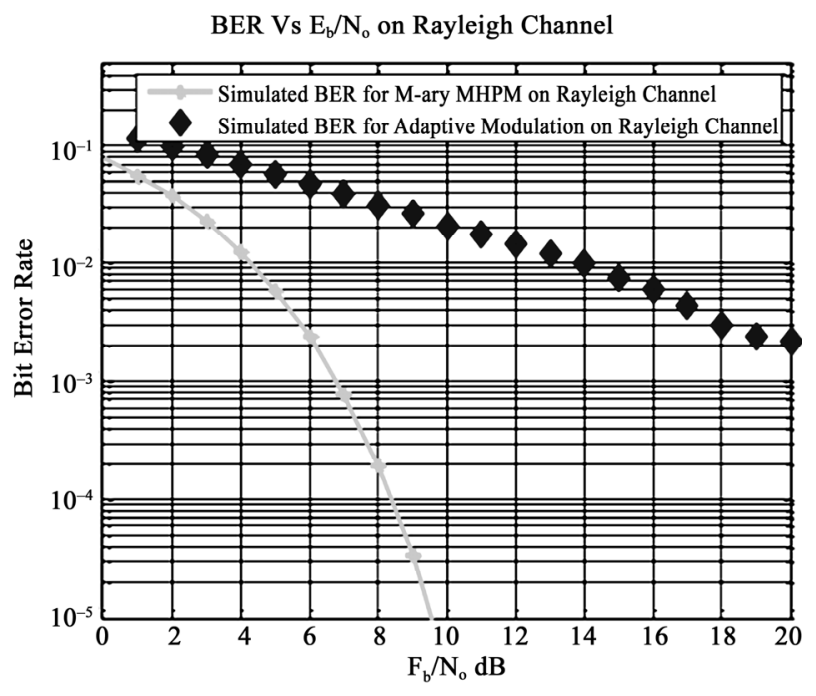

Figure 5. Comparison of overall performance of a system with adaptive modulated MC-CDMA system and M-ary MHPM in Rayleigh fading channel at CSNR $=15 \mathrm{~dB}$.

the bit rate per given bandwidth does not remain constant as in fixed modulation based systems but it varies according to the channel condition.

We found that the system using M-ary MHPM modulation technique shows the best performance among the considered modulation schemes. So an adaptive M-ary MHPM modulated MC-CDMA system is able to provide highly effective system in supporting high data rate services with mobility in wireless networks, and takes substantial care for the inter-symbol interference generated due to multipath effect and provides adequate bandwidth for the intended services.

\section{REFERENCES}

[1] J. K. Cavers, "Variable-Rate Transmission for Rayleigh Fading Channels," IEEE Transactions on Communication, Vol. 20, No. 1, 1972, pp. 15-22.

[2] S. Otsuki, S. Sampei and N. Morinaga, "Square-QAM Adaptive Modulation/TDMA/TDD Systems Using Modulation Level Estimation with Walsh Function," Electronics Letters, Vol. 31, No. 3, 1995, pp. 169-171.

[3] A. Goldsmith and S. G. Chua, "Adaptive Coded Modulation for Fading Channels," Transactions on Communication, Vol. 46, No. 5, 1998, pp. 595-602. doi:10.1109/26.668727

[4] GSM 02.60, "GPRS Service Description-Stage 1," Version 7.5.0, 1998.

[5] P. Stuckmann and O. Paul, "Dimensioning GSM/GRPS
Networks for Circuit-Switched and Packet-Switched Services," Proceedings of WPMC'01, Aalborg, Denmark, September 2001.

[6] H. Holma and A. Toskala, "WCDMA for UMTS-Radio Access for Third Generation Mobile Communications," John Wiley \& Sons, New York, 2001.

[7] 3GPP TS 25.211, "Physical Channels and Mapping of Transport Channels onto Physical Channels (FDD)," 2002.

[8] T. Ue, et al., "Symbol Rate and Modulation Level Controlled Adaptive Modulation/TDMA/TDD for High-BitRate Wireless Data Transmission," IEEE Transactions on Vehicular Technology, Vol. 47, No. 4, 1998, pp. 1134 1147. doi:10.1109/25.728485

[9] T.-S. Yang and A. Duel-Hallen, "Reliable Adaptive Modulation Aided by Observations of Another Fading Channel," IEEE Transactions on Communications, Vol. 52, No. 4, 2004.

[10] K. Miya, O. Kato, K. Homma, T. Kitade, M. Hayashi and T. Ue, "Wideband CDMA Systems in TDD-Mode Operation for IMT-2000," IEICE Transactions on Communications, Vol. E81-B, No. 8, 1998, pp. 1317-1326.

[11] A. Duel-Hallen, S. Hu and H. Hallen, "Long-Range Prediction of Fading Signals: Enabling Adaptive Transmission for Mobile Radio Channels," IEEE Signal Processing Magazine, Vol. 17, 2000, pp. 62-75.

[12] T. Eyceoz, A. Duel-Hallen and H. Hallen, "Deterministic Channel Modeling and Long-Range Prediction of FastFading Mobile Radio Channels," IEEE Communication Letters, Vol. 2, No. 9, 1998, pp. 254-256.

[13] T. Eyceoz, S. Hu and A. Duel-Hallen, "Performance Analysis of Longrange Prediction for Fast-Fading Channels," Proceedings of the 33rd Annual Conference on Information Sciences and Systems, Vol. 2, 1999, pp. 656- 661.

[14] A. Duel-Hallen, S. Hu and H. Hallen, "Long-Range Prediction of Fading Signals: Enabling Adaptive Transmission for Mobile Radio Channels," IEEE Signal Processing Magazine, Vol. 17, 2000, pp. 62-75.

[15] S. Falahati and A. Svensson, "Adaptive Modulation Systems for Predicted Wireless Channels," IEEE Transactions on Communication, Vol. 52, No. 2, 2004, pp. 307316.

[16] J. Pons and J. Dunlop, "Bit Error Rate Link Adaptation for GSM," The 9th IEEE International Symposium on Personal, Indoor and Mobile Radio Communications, Vol. 3, 8-11 September 1998, pp. 1530-1534.

[17] J. M. Torrence and L. Hanzo, "Upper Bound Performance of Adaptive Modulation in a Slow Rayleigh Fading Channel," IEEE Electronics Letters, Vol. 32, No. 8, 1996, pp. 718-719. 